\title{
Implementation of a Simplified State Estimator for Wind Turbine Monitoring on an Embedded System
}

\author{
Theis Bo Rasmussen, Guangya Yang \\ and Arne Hejde Nielsen \\ Department of Electrical Engineering \\ Center for Electric Power and Energy \\ Technical University of Denmark \\ 2800, Kgs. Lyngby, DK \\ Email: \{thras, gyy, ahn\}@elektro.dtu.dk
}

\author{
Zhao Yang Dong \\ School of Electrical Engineering and Telecommunications \\ University of New South Wales \\ New South Wales 2052, Australia
}

\begin{abstract}
The transition towards a cyber-physical energy system (CPES) entails an increased dependency on valid data. Simultaneously, an increasing implementation of renewable generation leads to possible control actions at individual distributed energy resources (DERs). A state estimation covering the whole system, including individual DER, is time consuming and numerically challenging. This paper presents the approach and results of implementing a simplified state estimator onto an embedded system for improving DER monitoring. The implemented state estimator is based on numerically robust orthogonal factorization and used on a set of state equations of a generic wind turbine generator (WTG). The simplified state estimator is tested by simulating a generic WTG model and evaluated based on its execution time and estimation accuracy. Results show its fast execution time, its accuracy in handling normal measurement error and its ability to provide reliable data in the case of gross errors in the set of measurements.
\end{abstract}

\section{INTRODUCTION}

T HE traditional power system is mainly composed of large centralized power plants, but since the turn of the century, countries worldwide have increased the integration of renewable energy sources (RES) [1]. At the same time, control methods utilizing distributed energy resources (DERs), to ensure a reliable delivery of electricity, have been proposed and included in grid codes [2], [3]. To manage the decentralization of control decisions, investments in advanced information and communication technology (ICT) infrastructure are made, increasing the data acquisition and improving the visibility of power system operation [4], [5].

Relying more on monitoring and control of DERs and having a more complicated technology mix on both sides of generation and consumption, the power system is transitioning into a cyber-physical energy system (CPES) [5], [6], [7], [8].

Historically, the process of state estimation has been used to remove measurement error within the boundaries of the power system [9], but within the larger and more complex CPES, centralized state estimation becomes computationally demanding and numerically challenging. Instead, the physical system at the boundaries of the power system could be observed and used for local state estimation purposes, removing gross measurements and assisting in the decision-making process of determining appropriate distributed control actions. The aim of this paper is to utilize this theory and implement a DER monitoring system onto an embedded system and determine its accuracy compared to that of raw measurements.

For this purpose, the generic wind turbine generator (WTG) model described in [10] is modelled in Simulink and analyzed with the purpose of developing a simplified state estimation model. Considering the limited resources of an embedded system, the simplified state estimator is implemented on a commercially available embedded system. In this work, the embedded system chosen is a National Instruments (NI) compact-RIO (cRIO) 9074.

In previous work of applying state estimation techniques to wind power plant monitoring [11], [12], the aim has been to investigate the dynamics of the WTGs, for testing and developing control designs, and improving the transient performance of WTGs. For these purposes, comprehensive dynamic models of WTGs are necessary to give the required level of detail. In this paper, the goal is to validate measurements in DER supervisory control and data acquisition (SCADA) systems to provide an accurate picture of the static operation of the DERs that can be utilized from a system operations perspective. Therefore, the accuracy and complexity of the WTG model can be decreased to enable execution of the simplified state estimator on an embedded system.

Results from testing the capability, accuracy and performance of the monitoring system, show that the state estimator is simple enough to be implemented onto an embedded system and execute within appropriate timing, is fairly accurate when normal measurement error is present and offers higher accuracy compared to the utilization of raw measurement data when gross measurement error is present.

The rest of the paper is organized as follows. In Section II the chosen state estimator algorithm is described, followed by a presentation of the derived state equations from the WTG Simulink model used in the state estimator. The section ends with a description of how the simplified state estimator was implemented on the embedded system using LabVIEW software. Section III presents the objective, analysis and evaluation of three test cases used to test the monitoring system. Section IV concludes this paper. 


\section{METHOD}

The concept of state estimation in power system application was presented in [9]. The purpose of the state estimation is to reduce measurement error e by estimating a set of state variables $\mathbf{x}$ related to the set of measurements $\mathbf{z}$ by a set of state equations $\mathbf{h}(\mathbf{x})$ as shown in Eq. (1).

$$
\mathbf{z}=\mathbf{h}(\mathbf{x})+\mathbf{e}
$$

Since the concept was introduced, numerous different algorithms have been proposed in literature, some of which are described in [13]. Most of these methods are based on the formulation of a set of non-linear equations, where the solution is found by solving a weighted least squares (WLS) problem [14]. The WLS problem is formulated as a optimization problem as described in (2).

$$
\operatorname{minimize} \quad J(\mathbf{x})=1 / 2 \sum_{j=1}^{m}\left(\frac{r_{j}^{2}}{\sigma_{j}^{2}}\right)
$$

where $J(\mathbf{x})$ is the weighted sum of square residuals, $m$ is the number of measurements, $r_{j}=z_{j}-h_{j}(\mathbf{x})$ is the residual and $\sigma_{j}^{2}$ is the variance of the $j$-th measurement. The variance of the measurements is based on the characteristics of the measurement devices. As measurement are devices are less than $100 \%$ accurate, it is assumed that its error is normal distributed with zero mean and variance $\sigma^{2}$ [14].

As the objective of this paper is to implement the state estimator on an embedded system, two requirements for the state estimation method are considered. The chosen methods have to 1) be numerically robust, as rounding errors are more likely in the embedded system compared to a control center computer, due to the limited bit number of the embedded operating system (OS) compared to general purpose OS, and 2) ensure an accurate convergence within the timing requirements set by the system.

The state estimation method used in this work is formulated around an iterative process where the updated state variables are calculated using orthogonal factorization, also referred to as QR factorization, which has been widely accepted in practice [14]. The stability of the factorization method comes from avoiding the formation of the gains matrix and thereby alleviating the numerically ill-conditioned state estimation problem. In [15] a comparative study has shown that the QR factorization is the most numerically robust, but at the same time has the highest computational requirements. To ensure convergence within the timing requirements, the complexity of the state equations, representing the WTG, is formulated from a trade-off between accuracy and computation requirements.

An added feature in power system state estimators, that improves the removal of measurement error, is the threefold process of bad data detection, identification and elimination that together form a bad data detector.

\section{A. Bad data detection, identification and elimination}

Mili, Van Cutsem and Ribbens-Pavella defined the task of the bad data detector, in the content of state estimation, as "Its task is to guarantee the reliability of the data base generated through the estimator." in [16, p.3037].

Bad data can occur in a monitoring system because of faulty measurement devices, faulty communication or even interference from adversaries [7]. In [17] measurement error has been characterized into three groups based on their magnitude compared to the standard deviation of the measurement device. Normal measurement error is expected to have a magnitude of up to $5 \sigma$, gross measurement error has a magnitude between $5 \sigma$ and $20 \sigma$, and extreme measurement error has a magnitude larger than $20 \sigma$.

There exist multiple different bad data detection algorithms in literature [16], [18], [19]. In this work, a simple bad data detector is implemented and designed to run after each iteration of the state estimation algorithm. The detection algorithm chosen is introduced in [9] and based on the concept of hypothesis testing and $J(\mathbf{x})$ tests. The method is based on an assumption that the weighted sum of square residuals, $J(\mathbf{x})$, follow a chi-square distribution, $\chi^{2}$, with a degree of freedom, $f$, equal to the number of measurements $m$ minus the number of state variables $n$.

By analyzing the chi square probability density function, $P$, a probability, $\alpha$, is chosen between $1 \%$ and $10 \%$ as a trade-off between the number of false positives and negatives [20] as indicated by Eq. (3).

$$
P\left[J(\mathbf{x})>K \mid J(\mathbf{x}) \sim \chi^{2}\right]=\alpha
$$

where the weighted sum of square residuals, $J(\mathbf{x})$ is calculated using Eq. (2). $K$ is characterized as the $(1-\alpha)$ quantile of the chi-square probability distribution with a degree of freedom equal to $(m-n)$ and is calculated using Eq. (4) [20].

$$
K=\chi_{(m-n): \alpha}^{2}
$$

The hypothesis of whether or not bad data is present in the set of measurements $\mathbf{z}$ is evaluated by comparing $J(\mathbf{x})$ to the detection threshold $K$ with a chosen $\alpha$-value. If $J(\mathbf{x})>K$ bad data is detected and vice versa. In the case of bad data being detected, the process of bad data identification is initiated. A widely used identification method of sorting the weighted residuals in $J(\mathbf{x})$ in a descending order and determining the measurement with the largest weighted residual as the bad measurement, is implemented in the bad data detector [18].

After detecting and identifying the bad data, the bad measurement must be eliminated to make sure the state estimator will converge towards an accurate solution. There exists multiple different techniques in eliminating bad data, with different computational requirements [16], [19]. As DERs are operating in a highly dynamic system, the process of simply replacing the bad data by the measurement from the last period is unreliable. Instead a similar approach as the one used in [19] is utilized, where the bad measurement is replaced 
by a pseudo measurement based on the estimated value and the gains matrix. In the simplified state estimator, the gains matrix is avoided, therefore the identified bad measurement is calculated using Eq. (5).

$$
z_{b}^{\text {new }}=z_{b}^{\text {old }}-\operatorname{sign}\left(z_{b}^{\text {old }}-h_{b}(\mathbf{x})\right) \cdot|a|
$$

where the subscript $b$ represents the index of the identified bad data, and $|a|$ represents the absolute value of a normal distributed random number with zero mean and a standard deviation of $\sigma=0.01$. The idea behind the value subtracted from the bad data to form the new data in Eq. (5), is that the sign of difference between the bad data and the estimated value is assumed to represent the sign of the difference between the bad data and the correct data. By simply pushing the bad data in the direction of the estimated value, the new data should be closer to the correct data, assuming the estimated value is closer to the correct data.

After safely eliminating the identified bad data, the state estimator executes its algorithm once again and the process of bad data detection, identification and elimination is repeated. It might be necessary to execute the bad data detector several times until the hypothesis of bad data being present is thrown.

\section{B. WTG state model}

The state estimator of the WTG generic model requires the three sets composing Eq. (1):

- A set of measurements $\mathbf{z}$

- A set of state variables $\mathbf{x}$

- A set of state equations $\mathbf{h}(\mathbf{x})$ relating the state variable to the measurements and the measurement error $\mathbf{e}$.

The SCADA system of a single wind turbine communicate more than 150 different values including temperature measurements, alarm state signals, and mechanical as well as electrical measurements of the wind turbine and the equipment connecting it to the collector system [21]. For the generic WTG model, 9 relevant mechanical and electrical properties are listed in Table I and used as inputs for the state estimation model.

TABLE I

WIND TURBINE SCADA MEASUREMENTS IN THE WTG STATE ESTIMATION MODEL

\begin{tabular}{|c|c|c|c|c|c|}
\hline \multicolumn{3}{|c|}{ Mechanical } & \multicolumn{3}{|c|}{ Electrical } \\
\hline Signal & Description & Unit & Signal & Description & Unit \\
\hline$V_{w}$ & Wind speed & {$[\mathrm{m} / \mathrm{s}]$} & $P$ & Active power & [W] \\
\hline$\theta$ & Pitch angle & {$\left[{ }^{\circ}\right]$} & $Q$ & Reactive power & [var] \\
\hline \multirow[t]{4}{*}{$\omega_{r}$} & Rotor speed & {$[\mathrm{pu}]$} & $U_{r m s}$ & Phase a rms voltage & {$[\mathrm{V}]$} \\
\hline & & & $I_{r m s}$ & Phase a rms current & {$[\mathrm{A}]$} \\
\hline & & & $U_{a}$ & Phase a voltage & {$[\mathrm{V}]$} \\
\hline & & & $I_{a}$ & Phase a current & {$[\mathrm{A}]$} \\
\hline
\end{tabular}

For the DER monitoring system implemented in this work, a measurement frequency of $1 \mathrm{~Hz}$ is chosen as it complies with the normal practice in SCADA systems [22], [23]. Simultaneously, this entails that the timing requirements of the state estimator is well below 1 second, as the embedded system has to acquire the measurement signals before executing the state estimator, and allow time for data communication and processing at control centers.

For all the measurements in Table I the normal measurement error is assumed to have zero mean and a standard deviation of $\sigma=0.01$, which corresponds to the measurement error introduced by measurement transformers for the electrical measurements and the errors entailed when measuring the mechanical system [24]. From the set of measurements in Table I, an appropriate set of state variables is identified. From state-space analysis theory [25], the state variables have to enable an estimation of all the input signals at any instance in time $t$. In the mechanical system, there is a relationship between the wind speed, the turbine rotational speed and the geometry of the wind turbine. If a steady wind is blowing, the tip speed ratio $\lambda$ defines this relationship through a constant $K_{b}$ as shown in Eq. (6).

$$
\lambda=\frac{K_{b} \cdot \omega_{r}}{V_{w}}
$$

For this project, the parameters given for a General Electric (GE) 1.5MW DFIG in [10] are used to represent the generic WTG. Due to the simplicity of the generic WTG model, a single mass model is used to represent the shaft connecting the rotor hub to the generator as recommended in [10].

The tip speed ratio can be used to estimate the pitch angle of the blades $\theta$. According to [26] the aerodynamic design of the wind turbine blades and their pitch angle has a certain relationship with the power coefficient $C_{p}(\theta, \lambda)$. These power coefficient curves are confidential and extremely difficult to access, therefore [10] has defined a relationship used in the generic GE 1.5MW DFIG WTG representation.

The built-in pitch controller of the generic WTG model attempts to maximize the power output according to the tip speed ratio. At very low wind speeds, the pitch controller keeps the pitch angle at $0^{\circ}$. When the wind speed increases, the pitch controller regulate the appropriate pitch angle in order to keep the power output at rated power. To get the relationship between tip speed ratio and pitch angle expressed as an equation, the WTG model is implemented in MATLAB Simulink, and simulated with a gradually increasing wind speed. Fig. 1 shows the resulting pitch angle as a function of tip speed ratio. At $\lambda>6$, the pitch angle is 0 .

From this discussion, it can be argued that from the wind speed and the rotational speed, it is possible to estimate the blade pitch angle. Therefore, the first two state variables of the state model are chosen as $x_{1}$ referring to $V_{w}$ and $x_{2}$ referring to $\omega_{r}$. In the electrical system, assuming availability of accurate voltage and current angles through phasor measurement units (PMU) [27], all the input signals can be estimated from the root-mean-square (rms) current and voltage. Therefore $x_{3}$ is chosen equal to $U_{r m s}$, likewise $x_{4}$ is chosen equal to $I_{r m s}$.

To improve the reliability of the state estimator, all the measurements are converted into the per unit (pu) scale, which decreases the differences in the non-zero elements of the 


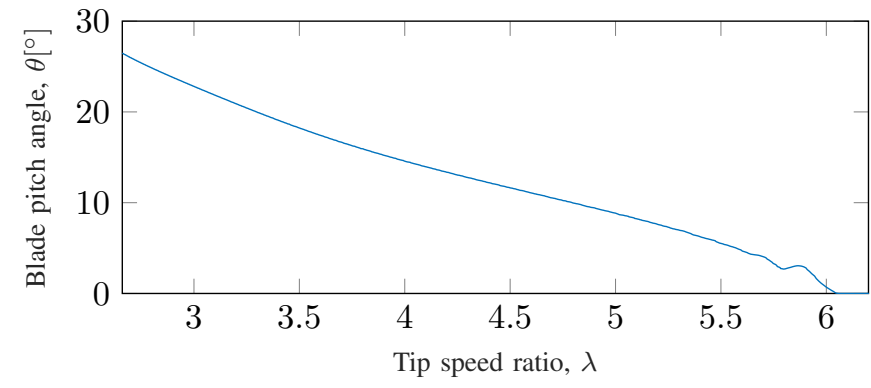

Fig. 1. Simulation results of the generic WTG model in MATLAB Simulink with graduate increasing wind speed, giving a relationship between pitch angle, $\theta$, and tip speed ratio, $\lambda$.

Jacobian matrix of the state equations. All measurements are converted into per unit using the base values shown in Table II.

TABLE II

PER UNIT BASE VALUES OF THE WTG MEASUREMENTS

\begin{tabular}{lc|lc|lc}
\hline \multicolumn{2}{c|}{ Mechanical } & \multicolumn{4}{c}{ Electrical } \\
Signal & Base value & Signal & Base value & Signal & Base value \\
\hline$V_{w}$ & $12 \mathrm{~m} / \mathrm{s}$ & $\mathrm{P}$ & $3 \mathrm{MVA}$ & $I_{r m s}$ & $2886.75 \mathrm{~A}$ \\
$\theta$ & $10.42^{\circ}$ & $\mathrm{Q}$ & $3 \mathrm{MVA}$ & $U_{a}$ & $346.41 \mathrm{~V}$ \\
$\omega_{r}$ & $1 \mathrm{pu}$ & $U_{r m s}$ & $346.41 \mathrm{~V}$ & $I_{a}$ & $2886.75 \mathrm{~A}$ \\
\hline
\end{tabular}

The per unit base values of the mechanical system are chosen based on the parameters of the GE 1.5MW WTG from [10], Eq. (6), and the relationship between $\lambda$ and $\theta$ illustrated in Fig. 1.

For the electrical system, the apparent power and voltage per unit base values are chosen based on the test system shown by the one line diagram in Fig. 2, created based on the benchmark tests performed in [10] and the cable data found in [28].

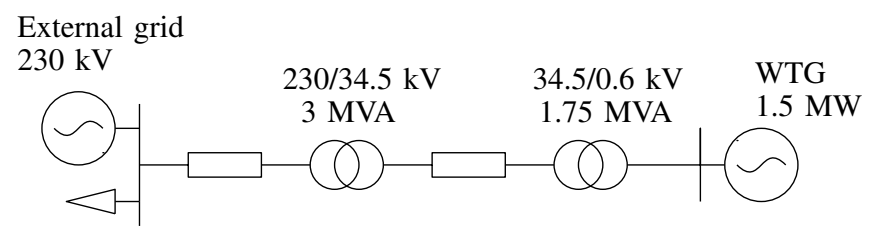

Fig. 2. Single line diagram of test grid used to simulate the WTG mode connected to an external grid.

The highest rated equipment is the $230 / 34.5 \mathrm{kV}$ transformer with an apparent power rating of 3 MVA. From the second transformer, it can be observed that the terminal voltage in line to line rms is $0.6 \mathrm{kV}$. Therefore the per unit values of the electrical system are calculated as in Table II.

After defining the per unit bases, the $\lambda, \theta$ relationship found in Fig. 1 is converted to per unit and analyzed through the MATLAB curve fitting tool to give the four term Gaussian function representing the state equation in Eq. (8).

$$
V_{w}=x_{1}
$$

$$
\begin{aligned}
\theta & =2.778 \cdot \exp \left(-\left(\frac{\frac{x_{2}}{x_{1}}-0.4469}{0.3078}\right)^{2}\right) \\
& +0.8212 \cdot \exp \left(-\left(\frac{\frac{x_{2}}{x_{1}}-0.8423}{0.2008}\right)^{2}\right) \\
& +0.4885 \cdot \exp \left(-\left(\frac{\frac{x_{2}}{x_{1}}-1.037}{0.1354}\right)^{2}\right) \\
& +0.2784 \cdot \exp \left(-\left(\frac{\frac{x_{2}}{x_{1}}-1.169}{0.08579}\right)^{2}\right) \\
\omega_{r} & =x_{2} \\
P & =x_{3} \cdot x_{4} \cdot \cos (\phi) \\
Q & =x_{3} \cdot x_{4} \cdot \sin (\phi) \\
U_{r m s} & =x_{3} \\
I_{r m s} & =x_{4} \\
U & =x_{3} \cdot \sqrt{2} \cdot \sin (2 \pi f t+\delta) \\
I & =x_{4} \cdot \sqrt{2} \cdot \sin (2 \pi f t+\beta)
\end{aligned}
$$

The equations in Eq. (7) to (9) are the mechanical state equations and together with Eq. (10) to (15), they form the complete set of state equations $\mathbf{h}(\mathbf{x})$. The set of electrical state equations, in Eq. (10) to (15), are found from power system theory [29].

\section{LabVIEW implementation}

The DER monitoring system is tested by implementing a simulation model of the WTG onto the cRIO through the LabVIEW programming tool. The Simulink WTG model is built as a C code, using the compiler in Simulink, and implemented on the cRIO through the model interface toolkit (MIT) in LabVIEW.

The added computational burden on the cRIO is considered by lowering the simulation of the WTG model. To include necessary details of voltage and current waveforms from the WTG model, the simulation frequency is set to $2500 \mathrm{~Hz}$. On the cRIO, each simulation step will be executed 25 times slower than real time, this will however not affect the execution time evaluation of the state estimator.

With the Simulink model implemented on the cRIO, the simplified state estimator and integrated bad data detector are programmed in LabVIEW, as described in Section II and II-A, and shown in the process diagram in Fig. 3, which contains additional information about the inter-process and inter-target communication.

To allow control of the wind speed, a real-time (RT) target process is created to simulate wind speed according to the model described in [30]. The wind speed $V_{w}$ and WTG simulation model are executed in synchronized while loops on the RT target to make sure that the calculations are executed in a deterministic fashion. Before executing the state estimator in the process of Fig. 3, each Simulink signal is distorted by a normal distributed measurement error with zero mean and variance equal to $\sigma^{2}=10^{-4}$. After completing an iteration of the state estimation process, the updated state variables are 
System Diagram cRIO

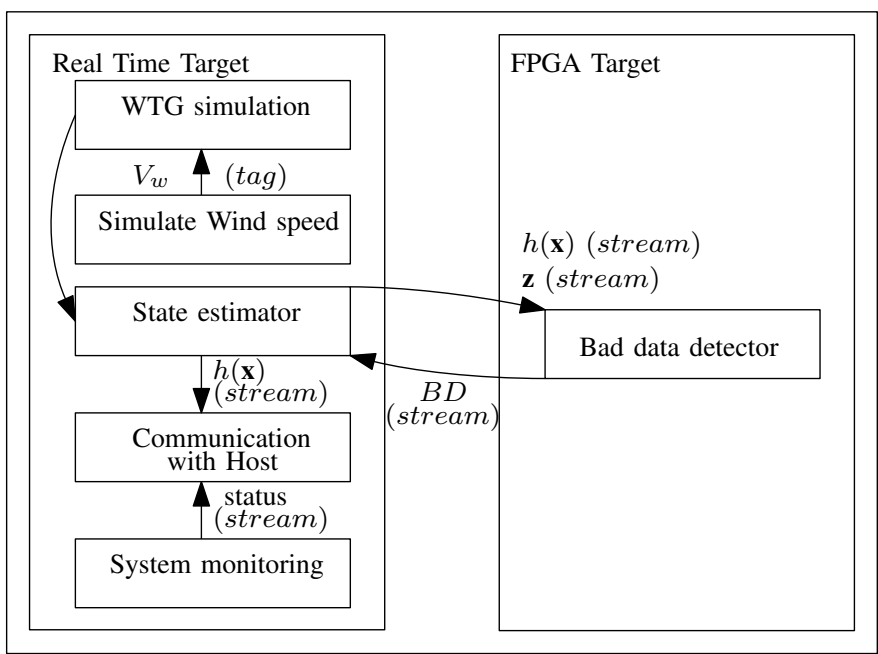

Fig. 3. System diagram of the cRIO with the Simulink model of the generic WTG implemented in LabVIEW.

communicated to the field-programmable gate array (FPGA) target where bad data is detected and identified.

The information about bad data is returned to the state estimator, which eliminates the bad measurements through Eq. (5). When the Euclidean norm of the state variables have converged below the tolerance $x_{\text {convergence }}$ and no bad data is detected, the information is returned to the host and visualized for the system operator.

\section{RESULTS}

To evaluate the application of the embedded system for DER monitoring the following three factors are considered:

1) Its ability to solve the WLS problem within a short time frame.

2) Its accuracy in estimating the solution to the state estimation model compared to raw measurements when subject to normal measurement error.

3) Its performance in terms of detecting, identifying and eliminating gross measurements errors.

Each factor is evaluated through a test scenario. In the following, three test cases are introduced, the results are analyzed and the system is evaluated.

\section{A. Test 1: Execution time}

The purpose of the first test is to evaluate how fast the simplified state estimator with integrated bad data detector can solve the WLS problem. This objective is reached by implementing tick counts in the LabVIEW data flow before and after the state estimator and bad data detector process in Fig. 3.

Under normal conditions, with a standard deviation equal to the assumed measurement device accuracy of $\sigma=0.01$, the QR factorization algorithm only requires a single or two iterations to solve the WLS problem. To evaluate the execution time of the DER monitoring system at different number of

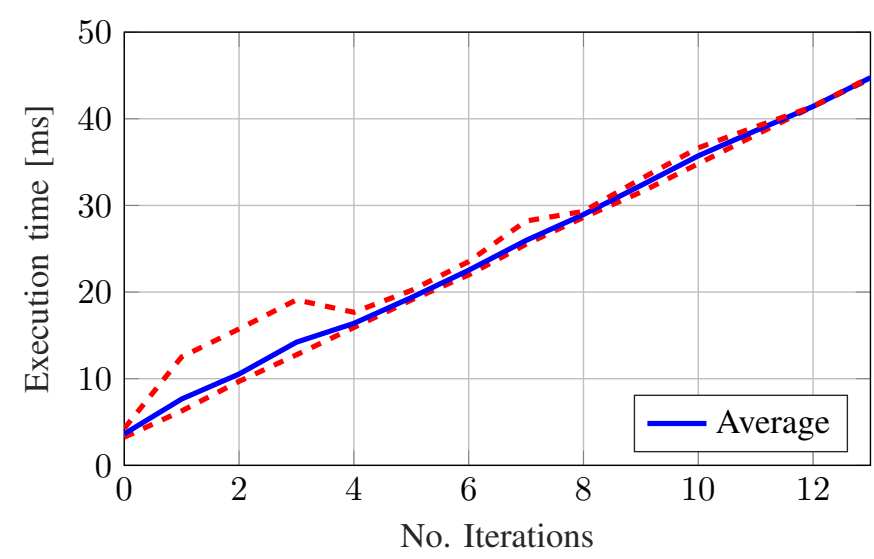

Fig. 4. Average, minimum and maximum execution time of the state estimator, with integrated bad data detector, as a function of the number of iterations needed before converging.

iterations, the standard deviation of the measurement error is increased to $\sigma=0.02$. This results in a higher chance of the measurement error causing a detection of bad data, while using a detection probability of $\alpha=5 \%$, and thereby increases the number of iterations needed to solve the WLS problem.

The cRIO is run for a time series where the state estimator executes in total 162 times. The resulting execution time data is separated based on the number of iterations needed before finding a solution to the WLS problem, reached after the Euclidean norm of the change in state variable value between two iterations is below the convergence threshold chosen as $x_{\text {converge }}=0.01$.

The number of iterations ranges from 0 to 13 . In the case where no iterations are needed, the first solution of the state estimator is close enough to the final solution of the previous execution, used as the starting point for the following execution. The minimum, average and maximum execution time is calculated and presented in Fig. 4 as a function of the required number of iterations before converging.

A linear relationship between the number of iterations and the average execution time is observed in Fig. 4. For the executions with 1 to 3 executions, the maximum observed execution is around $5 \mathrm{~ms}$ slower than the average execution time. At the same time, the average value is observed closer to the minimum execution time, which indicates that the occurrence of large execution times is rather limited.

From Fig. 4 the execution time of the embedded DER monitoring system can be evaluated. As previously mentioned, the system is intended to run between acquisition and communication of data, and the added timing requirements of validating the data should be low enough to allow further data handling. For an iteration count between 0 and 13, the execution time varies from around $5 \mathrm{~ms}$ to $45 \mathrm{~ms}$.

Considering the case of two iterations, the average execution time is calculated in Fig. 4 as approximately $10 \mathrm{~ms}$, this corresponds to an execution frequency of $100 \mathrm{~Hz}$. An execution frequency of $100 \mathrm{~Hz}$ satisfies current SCADA requirements 

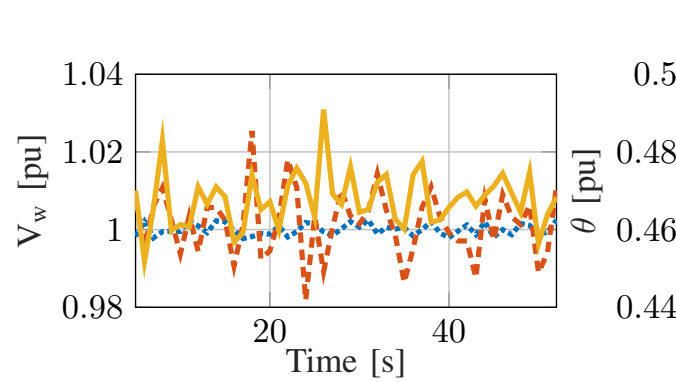

$\ldots \ldots \mathrm{Z}=\ldots=\mathrm{Z} \mathrm{d}=\mathrm{h}(\mathrm{x})$
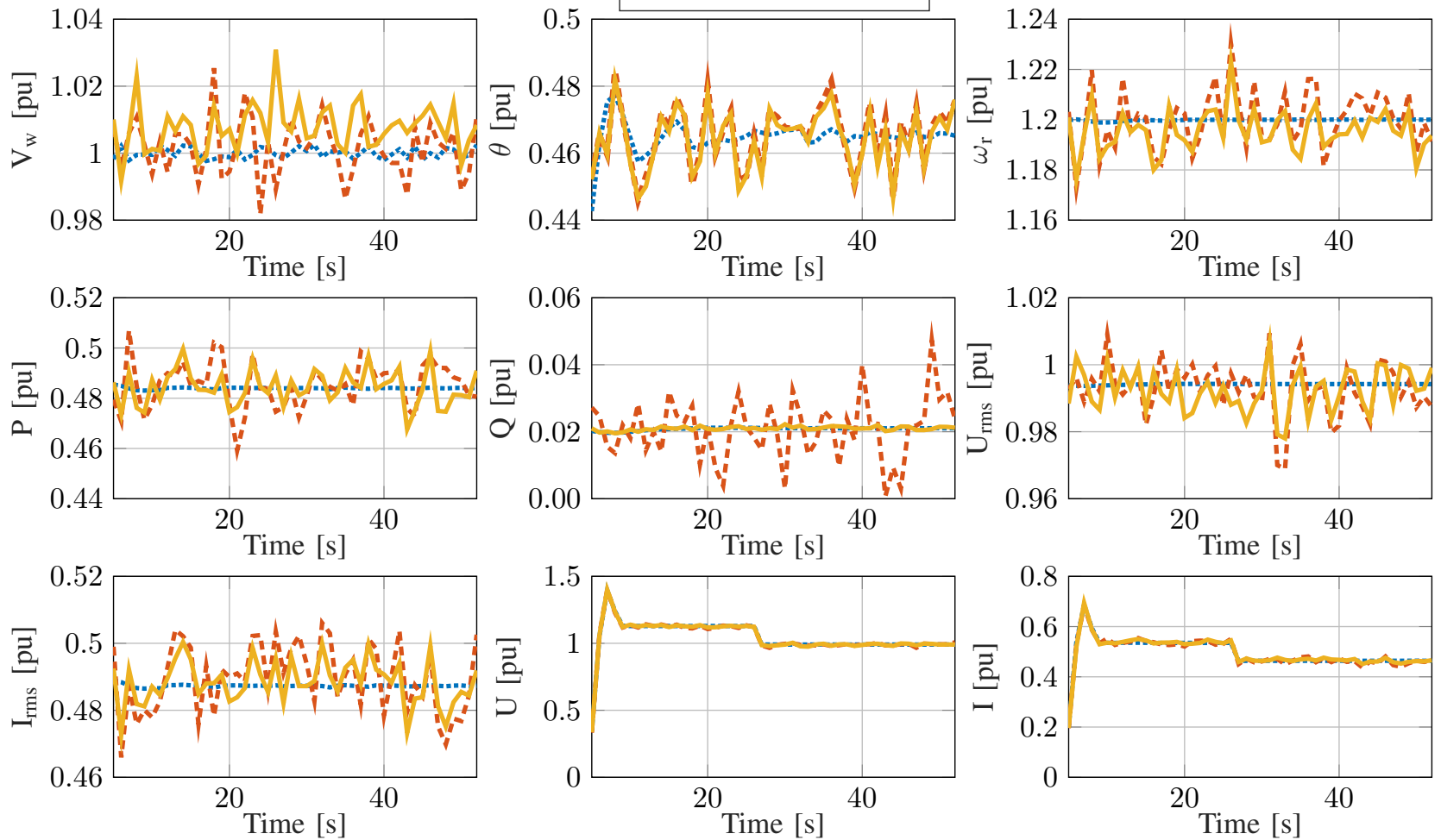

Fig. 5. The value of the nine measurements in $\mathbf{z}$, represented by the true simulated signal (blue line), the distorted signal (red line) and the estimated signal (yellow line).

and offers possibilities in terms of allowing a faster data acquisition for future SCADA systems.

\section{B. Test 2: Estimation accuracy}

In the second test, the objective is to compare the accuracy of the estimated and disturbed signals to the correct signals from the Simulink model. In this test case, all measurements are disturbed by normal measurement error with zero mean and $\sigma=0.01$.

The cRIO is run and the accuracy of the simplified state estimator is analysed by comparing the estimated signals $\mathbf{h}(\mathbf{x})$ to the correct simulated signals $\mathbf{z}$ and the distorted signals $\mathbf{z}_{d}$. These three results are found for each measurement in Table I represented by their per unit value corresponding to Table II, and shown in Fig. 5.

From the nine plots in Fig. 5 the dynamics of the system are observed from changes in $\mathbf{z}$ during the time period. This is especially visible for the wind speed $V_{w}$, the blade pitch angle $\theta$ and the instantaneous voltage $U$ and current $I$. For all the measurements, $\mathbf{h}(\mathbf{x})$ is closer to or similarly distanced from $\mathbf{z}$ compared to the disturbed measurements $\mathbf{z}_{d}$.

A numerical comparison of the results in Fig. 5 is performed by calculating the average Euclidean error (AEE) over the executed time period $\tau$, using Eq. (16), as introduced in [31].

$$
A E E\left(d_{i}\right)=\frac{1}{\tau} \sum_{t=1}^{\tau}\left\|d_{t, i}\right\|_{2}
$$

where $\mathbf{d}=\mathbf{z}-\mathbf{v}, \mathbf{v}$ is a set of values who's difference from the correct measurements is desired, and $i$ is the index of the measurements in Table I. The AEE is calculated for both $\mathbf{z}_{d}$ and $\mathbf{h}(\mathbf{x})$ and is shown in Table III.

TABLE III

AVERAGE EUCLIDEAN ERROR OF ESTIMATED AND DISTURBED VALUES FOR THE TIME SERIES RESULTS IN FIG. 5

\begin{tabular}{|c|c|c|c|}
\hline \multicolumn{2}{|c|}{$\mathbf{v}=$} & $\mathbf{z}_{d}$ & $\mathbf{h}(\mathbf{x})$ \\
\hline \multirow{9}{*}{$\operatorname{AEE}\left(d_{i}\right)$} & $i=1$ & 0.0067 & 0.0085 \\
\hline & $i=2$ & 0.0067 & 0.0064 \\
\hline & $i=3$ & 0.0095 & 0.0074 \\
\hline & $i=4$ & 0.0074 & 0.0055 \\
\hline & $i=5$ & 0.0070 & 0.0004 \\
\hline & $i=6$ & 0.0061 & 0.0057 \\
\hline & $i=7$ & 0.0090 & 0.0052 \\
\hline & $i=8$ & 0.0084 & 0.0056 \\
\hline & $i=9$ & 0.0085 & 0.0053 \\
\hline
\end{tabular}

The small values of all the AEE results in Table III show the similarity of the average error of $\mathbf{z}_{d}$ and $\mathbf{h}(\mathbf{x})$. Evaluating the accuracy of the simplified state estimator based on these results gives an indication that $\mathbf{h}(\mathbf{x})$ offers similar accuracy in situations with normal measurement noise as the raw measurements. The state estimator could be improved by utilizing a more detailed set of state equations as in [11] or [12], however this would simultaneously change the execution time as the 

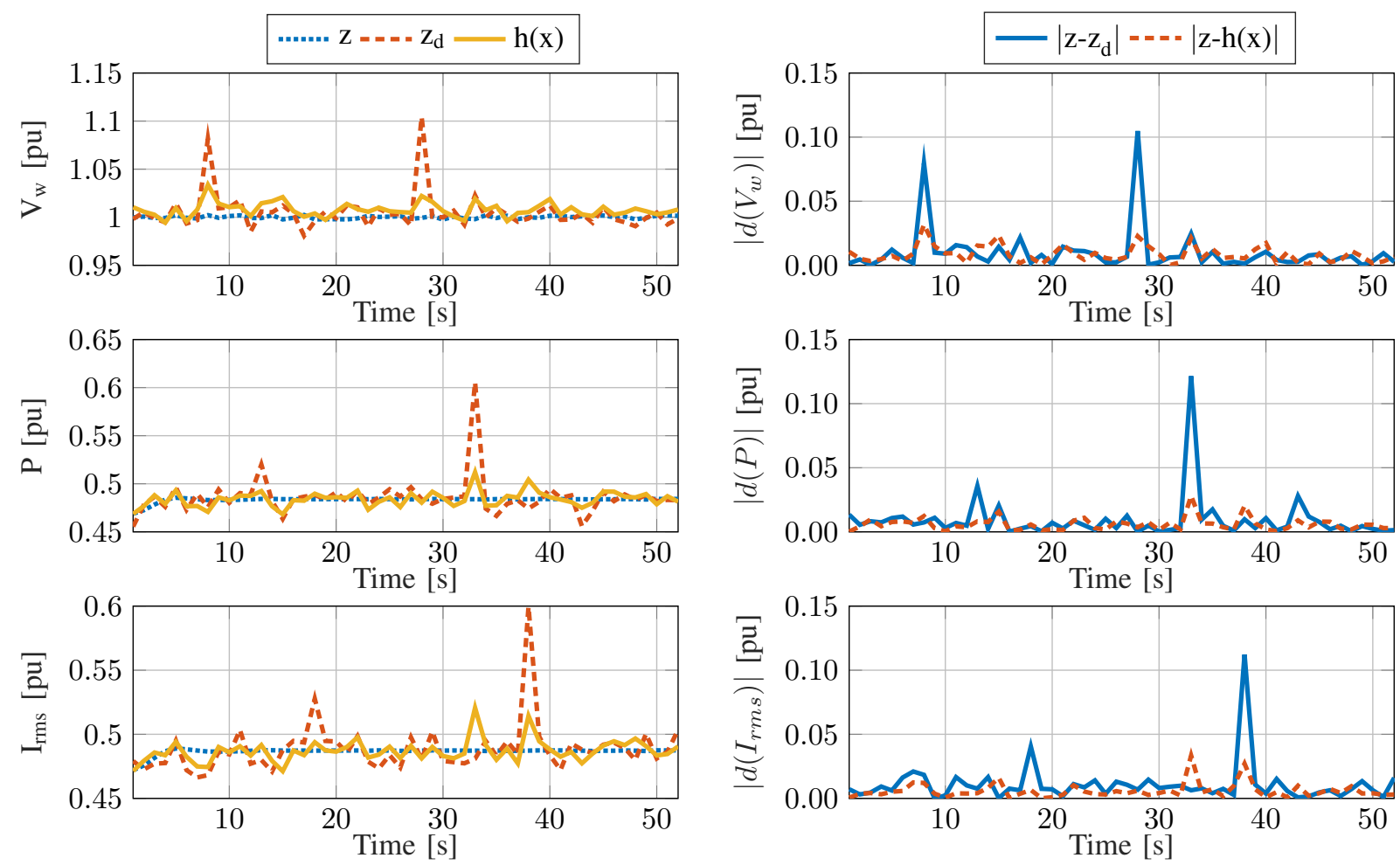

Fig. 6. Left: The wind speed, active power and rms current in per unit when subject to gross measurement error. Right: The absolute error between the true simulated signal and the distorted signal (blue line) and the estimated signal (red line), for the three measurements subject to gross error.

detailed model requires an increased number of calculations in finding the solution to the WLS problem.

\section{Test 3: Gross error performance}

After testing the accuracy of the state estimators when measurements are subject to normal measurement error only, this test case evaluates the performance of the embedded DER monitoring system when gross measurement errors are injected into a set of target measurements. For this purpose, a testing interface is implemented in the LabVIEW user interface that allows specification of scalar measurement error and the index of the target measurement.

Three different measurements are chosen as targets and injected with the gross measurement error $\varepsilon$ at the time $t_{\varepsilon}$ as presented in Table IV.

TABLE IV

GROSS MEASUREMENT ERROR INJECTION SCHEDULE FOR TEST CASE 3.

\begin{tabular}{c|cccccc}
\hline$t_{\varepsilon}$ & $8 \mathrm{~s}$ & $13 \mathrm{~s}$ & $18 \mathrm{~s}$ & $38 \mathrm{~s}$ & $33 \mathrm{~s}$ & $38 \mathrm{~s}$ \\
\hline$z_{i}$ & $V_{w}$ & $P$ & $I_{r m s}$ & $V_{w}$ & $P$ & $I_{r m s}$ \\
$\varepsilon$ & $5 \sigma$ & $5 \sigma$ & $5 \sigma$ & $10 \sigma$ & $10 \sigma$ & $10 \sigma$ \\
\hline
\end{tabular}

From Table IV the magnitude of the gross measurement error injected is chosen as 5 and 10 times the standard deviation of all the measurements $\sigma=0.01$. The schedule is used while running the cRIO, giving the results illustrated in Fig. 6.
In Fig. 6, the left hand side shows the pu value of the wind speed, the active power and the rms current during the time period of execution. From these plots, $\mathbf{z}_{d}$ is clearly affected by the gross measurement error injected two times for each measurement. In comparison to $\mathbf{z}_{d}$, the estimated results in $\mathbf{h}(\mathbf{x})$ are closer to the correct measurements, $\mathbf{z}$ for each injection of gross measurement error.

In the right hand side plot of Fig. 6, the absolute error between $\mathbf{z}, \mathbf{z}_{d}$ and $\mathbf{h}(\mathbf{x})$ is shown for each of the three measurements. Here the performance, of the simplified state estimator, in handling gross measurement errors is easily visible, as it is able to detector, identify and eliminate the error and estimate a better signal value than the raw measurements.

The error in $I_{r m s}$ after $33 \mathrm{~s}$ in the right hand side of Fig. 6, equal to approximately $0.04 \mathrm{pu}$ indicates room for further improvements of the system. The cause of the large error is that the embedded monitoring system first correctly identifies the active power as the bad measurement, and after eliminating the error, it wrongly identifies a bad data at the rms current as well. This increases the difference between the pseudo measurement value and the correct value. This could possibly be avoided by finding the optimal trade-off between false positives and negatives, thereby fine tuning the detection threshold $K$, or refining the methods used in the bad data detector.

Besides the false identification of the rms current as containing a bad data, the results confirm the added accuracy of using the embedded DER monitoring system compared to 
using raw measurements when monitoring the performance of DERs. This accuracy could be valuable when considering the utilization of measurements in determining control actions in the CPES.

\section{CONCLUSION}

The growing implementation of distributed energy resources and the increased focus on distributed control of these resources entails added challenges in the cyber-physical energy system. With the added dependency on distributed control comes dependency on valid data from distributed energy resource measurement systems.

This paper describes the development, implementation and testing of a simplified state estimator, capable of efficiently removing gross measurement errors from distributed energy resource data measurements. The simplified state estimator is implemented on an embedded system and simulated in connection to a simulation model of a generic wind turbine generator. With the embedded system implementation, the measurements from the distributed energy resources can be processed and validated between data acquisition and data communication.

Simulation results show that the simplified state estimator has a fast execution time which offers utilization in current and future measurement systems. Compared to utilizing raw measurement data, the simplified state estimator has similar average Euclidean error as normal measurement error and can remove gross measurements, which shows its application potential in the cyber-physical energy system.

For future work, the bad data detector of the embedded monitoring system could be improved in terms of its ability to accurately identify bad data. A second proposed further research could be to try and validate the efficiency of the simplified state estimator by using real wind turbine measurements, and in the end, try to implement the system on a real wind turbine. A third possibility is to test the generality of the monitoring system by replacing the WTG state estimation model and applying the system on a different type of, such as a photovoltaic system.

\section{REFERENCES}

[1] P. Beiter and T. Tian, "2015 renewable energy data book," U.S. Department of Energy's National Renewable Energy Laboratory (NREL), Tech. Rep., 2016.

[2] Energinet.dk, "Technical regulation 3.2 .5 for wind power plants above 11 kw," Energinet.dk, Tech. Rep. 13/96336-43, 2016.

[3] - "Technical regulation 3.2.2 for pv power plants above $11 \mathrm{kw}$," Energinet.dk, Tech. Rep. 14/17997-39, 2016.

[4] F. F. Wu, K. Moslehi, and A. Bose, "Power system control centers Past, present, and future," Proceedings of the IEEE, vol. 93, no. 11, pp. 1890-1908, Nov 2005. http://dx.doi.org/10.1109/JPROC.2005.857499.

[5] X. Yu and Y. Xue, "Smart grids: A cyber-physical systems perspective," Proc. IEEE, vol. 104, no. 5, pp. 1058-1070, May 2016. http://dx.doi.org/10.1109/JPROC.2015.2503119.

[6] R. Rajkumar, I. Lee, L. Sha, and J. Stankovic, "Cyber-physical systems: The next computing revolution," in Design Automation Conference, June 2010, pp. 731-736. http://dx.doi.org/10.1145/1837274.1837461.

[7] S. Sridhar, A. Hahn, and M. Govindarasu, "Cyber-physical system security for the electric power grid," Proceedings of the IEEE, vol. 100, no. 1, pp. 210-224, Jan 2012. http://dx.doi.org/10.1109/JPROC.2011.2165269.
[8] X. Shi, Y. Li, Y. Cao, and Y. Tan, "Cyber-physical electrical energy systems: challenges and issues," CSEE Journal of Power and Energy Systems, vol. 1, no. 2, pp. 36-42, June 2015. http://dx.doi.org/10.17775/CSEEJPES.2015.00017.

[9] F. C. Schweppe, J. Wildes, and D. B. Rom, "Power system staticstate estimation, parts I, II, III," IEEE Transactions on Power Apparatus and Systems, vol. PAS-89, no. 1, pp. 120-135, Jan 1970. http://dx.doi.org/10.1109/TPAS.1970.292678.

[10] K. Clark, N. W. Miller, and J. J. Sanchez-Gasca, "Modeling of GE wind turbine-generators for grid studies," GE Energy, Tech. Rep. Version 4.4, September 2009.

[11] S. Yu, K. Emami, T. Fernando, H. H. C. Iu, and K. P. Wong, "State estimation of doubly fed induction generator wind turbine in complex power systems," IEEE Transactions on Power Systems, vol. 31, no. 6, pp. 49354944, Nov 2016. http://dx.doi.org/10.1109/TPWRS.2015.2507620.

[12] S. A. A. Shahriari, M. Raoofat, M. Dehghani, M. Mohammadi, and M. Saad, "Dynamic state estimation of a permanent magnet synchronous generator-based wind turbine," IET Renewable Power Generation, vol. 10, no. 9, pp. 1278-1286, 2018. http://dx.doi.org/10.1049/ietrpg.2015.0502.

[13] F. F. Wu, "Power system state estimation: a survey," International Journal of Electrical Power \& Energy Systems, vol. 12, no. 2, pp. $80-$ 87, 1990. http://dx.doi.org/10.1016/0142-0615(90)90003-T

[14] A. Monticelli, "Electric power system state estimation," Proceed ings of the IEEE, vol. 88, no. 2, pp. 262-282, Feb 2000. http://dx.doi.org/10.1109/5.824004.

[15] L. Holten, A. Gjelsvik, S. Aam, F. F. Wu, and W. H. E. Liu, "Comparison of different methods for state estimation," IEEE Transactions on Power Systems, vol. 3, no. 4, pp. 1798-1806, Nov 1988. http://dx.doi.org/10.1109/59.192998.

[16] L. Mili, T. V. Cutsem, and M. R.-P. and, "Bad data identification methods in power system state estimation-a comparative study," IEEE Transactions on Power Apparatus and Systems, vol. PAS-104, no. 11, pp. 3037-3049, Nov 1985. http://dx.doi.org/10.1109/TPAS.1985.318945.

[17] H. J. Koglin, T. Neisius, G. Beißler, and K. D. Schmitt, "Bad data detection and identification," International Journal of Electrical Power \& Energy Systems, vol. 12, no. 2, pp. 94-103, 1990. http://dx.doi.org/10.1016/0142-0615(90)90005-V.

[18] E. Handschin, F. C. Schweppe, J. Kohlas, and A. Fiechter, "Bad data analysis for power system state estimation," IEEE Transactions on Power Apparatus and Systems, vol. 94, no. 2, pp. 329-337, Mar 1975. http://dx.doi.org/10.1109/TPAS.1975.31858.

[19] A. Garcia, A. Monticelli, and P. Abreu, "Fast decoupled state estimation and bad data processing," IEEE Transactions on Power Apparatus and Systems, vol. PAS-98, no. 5, pp. 1645-1652, Sept 1979. http://dx.doi.org/10.1109/TPAS.1979.319482.

[20] V. H. Quintana, A. Simoes-Costa, and M. Mier, "Bad data detection and identification techniques using estimation orthogonal methods," IEEE Transactions on Power Apparatus and Systems, vol. PAS-101, no. 9, pp. 3356-3364, Sept 1982. http://dx.doi.org/10.1109/TPAS.1982.317595.

[21] M. Schlechtingen, I. F. Santos, and S. Achiche, "Wind turbine condition monitoring based on SCADA data using normal behavior models. part 1: System description," Applied Soft Computing, vol. 13, no. 1, pp. 259 - 270, 2013. http://dx.doi.org/10.1016/j.asoc.2012.08.033.

[22] J. R. Kristoffersen and P. Christiansen, "Horns Rev offshore windfarm: its main controller and remote control system," Wind Engineering, vol. 27, no. 5, pp. 351 - 360, 2003. http://dx.doi.org/10.1260/030952403322770959.

[23] B. Badrzadeh, M. Bradt, N. Castillo, R. Janakiraman, R. Kennedy, S. Klein, T. Smith, and L. Vargas, "Wind power plant scada and controls," in PES T D 2012, May 2012, pp. 1-7. http://dx.doi.org/10.1109/PES.2011.6039418.

[24] A. Ellis, Y. Kazachkov, J. Sanchez-Gasca, p. Pourbeik, E. Muljadi, M. Behnke, J. Fortmann, and S. Seman, Wind Power in Power Systems. John Wiley \& Sons, Ltd., 2012, ch. 35: A Generic Wind Power Plant Model. ISBN: 9780470974162.

[25] B. P. Lathi, Signal Processing and Linear Systems, international ed. ed. Oxford, United Kingdom: Oxford University Press, 2010. ISBN: 9780-19-539257-9.

[26] A. D. Hansen, P. Sørensen, F. Iov, and F. Blaabjerg, "Control of variable speed wind turbines with doubly-fed induction generators," Wind Engineering, vol. 28, no. 4, pp. 411-432, 2004. http://dx.doi.org/10.1260/0309524042886441 
[27] K. E. Martin, "Synchrophasor measurements under the ieee standard c37.118.1-2011 with amendment c37.118.1a," IEEE Transactions on Power Delivery, vol. 30, no. 3, pp. 1514-1522, June 2015 http://dx.doi.org/10.1109/TPWRD.2015.2403591.

[28] ABB, "XLPE submarine cable systems attachment to XLPE land cable systems - user's guide," Brochure, April 2010, rev. 5.

[29] J. D. Glover, M. S. Sarma, and T. J. Overbye, Power System Analysis and Design, 5th ed. Stamford, CT: Cengage Learning, 2008. ISBN: 978-
1-111-42579-1.

[30] P. M. Anderson and A. Bose, "Stability simulation of wind turbine systems," IEEE Transactions on Power Apparatus and Systems, vol. PAS-102, no. 12, pp. 3791-3795, Dec 1983. http://dx.doi.org/10.1109/TPAS.1983.317873.

[31] X. R. Li and Z. Zhao, "Measures of performance for evaluation of estimators and filters," vol. 4473, 2001, pp. 530-541. http://dx.doi.org/10.1117/12.492751. 